\title{
Finite-temperature properties of $\mathrm{Pb}\left(\mathrm{Zr}_{1-x} \mathrm{Ti}_{x}\right) \mathrm{O}_{3}$ alloys from first principles
}

\author{
L. Bellaiche ${ }^{1}$, Alberto Garcia ${ }^{2}$ and David Vanderbilt ${ }^{3}$ \\ 1 Physics Department, \\ University of Arkansas, Fayetteville, Arkansas 72701, USA \\ ${ }^{2}$ Departamento de Fisica Aplicada II, \\ Universidad del Pais Vasco, Apartado 644, 48080 Bilbao, Spain \\ 3 Center for Materials Theory, Department of Physics and Astronomy, \\ Rutgers University, Piscataway, New Jersey 08855-0849, USA
}

(April 21, 2000)

\begin{abstract}
A first-principles-derived approach is developed to study finite-temperature properties of $\mathrm{Pb}\left(\mathrm{Zr}_{1-x} \mathrm{Ti}_{x}\right) \mathrm{O}_{3}(\mathrm{PZT})$ solid solutions near the morphotropic phase boundary (MPB). Structural and piezoelectric predictions are in excellent agreement with experimental data and direct firstprinciples results. A low-temperature monoclinic phase is confirmed to exist, and is demonstrated to act as a bridge between the well-known tetragonal and rhombohedral phases delimiting the MPB. A successful explanation for the large piezoelectricity found in PZT ceramics is also provided.
\end{abstract}

PACS:77.84.Dy,81.30.Bx,77.65.Bn

Ferroelectric perovskite $\mathrm{A}\left(\mathrm{B}^{\prime} \mathrm{B}^{\prime \prime}\right) \mathrm{O}_{3}$ alloys are of growing importance for a variety of device applications [1,2, and are also of great current fundamental interest since little is known about the effects responsible for their anomalous properties. A good example of an $\mathrm{A}\left(\mathrm{B}^{\prime}, \mathrm{B}^{\prime \prime}\right) \mathrm{O}_{3}$ solid solution that is of both fundamental and technological importance is the $\mathrm{Pb}\left(\mathrm{Zr}_{1-x} \mathrm{Ti}_{x}\right) \mathrm{O}_{3}$ system. Usually denoted as PZT, this mixed-cation alloy is currently in widespread use in piezoelectric transducers and actuators [1]. Its phase diagram exhibits a morphotropic phase boundary (MPB) separating a region with a tetragonal ground state $(x>0.52)$ from a region with rhombohedral symmetry $(x<0.45)[3]$.

High piezoelectric response is experimentally found in ceramics of PZT around the MPB. The origins of this large piezoelectric response are unclear. On the one hand, semi-empirical simulations predict that the large experimental value of the $d_{33}$ piezoelectric coefficient results mainly from the large value of $d_{33}$ that a single-crystal PZT would exhibit [1]. On the other hand, recent firstprinciples calculations [5. [6] have found that the $d_{33}$ coefficient of a tetragonal single crystal of $\mathrm{Pb}\left(\mathrm{Zr}_{0.5} \mathrm{Ti}_{0.5}\right) \mathrm{O}_{3}$ are estimated to be three times smaller than the experimental value obtained for ceramics at low temperature.

Furthermore, recent synchrotron x-ray powder diffraction studies have revealed the existence of an unexpected low-temperature monoclinic phase of PZT at $x=0.48$ [7], which implies that the phase diagram of PZT is more complex than previously thought. This monoclinic phase may act as a second-order transitional bridge between the tetragonal phase, for which the electrical polarization $\mathbf{P}$ lies along the pseudo-cubic [001] direction, and the rhombohedral phase, for which $\mathbf{P}$ is along the pseudo-cubic [111] direction. If this is indeed the case, the polarization of the monoclinic phase continuously rotates as the composition $x$ decreases in the MPB region [7]. Such a continuous rotation has yet to be observed.
Obviously, accurate simulations are needed to understand the properties of perovskite alloys in general, and of PZT in particular. Since the beginning of the present decade, first-principles methods have emerged as a powerful tool for investigating properties of ferroelectric systems theoretically (see [5,6,8,8,9 and references therein). However, these methods are essentially restricted to the study of the zero-temperature properties of small cells, while accurate and interesting predictions of alloy properties would require calculations on much larger cells at finite temperature. Ideally one desires a computational scheme with the capability of predicting the properties of "real" perovskite alloy systems at finite temperature, with the accuracy of the first-principles methods.

The purpose of this letter is to demonstrate that it is possible to develop such a scheme, and to apply it to study the finite-temperature behavior of PZT in the vicinity of the MPB. Remarkably, we find that the existence of an intermediate monoclinic phase emerges naturally from this approach. Moreover, the theory provides a novel and successful explanation for the large piezoelectric response of PZT near the MPB, thereby explaining and resolving the previous theoretical difficulties in obtaining agreement with the known experimental values of the piezoelectric coefficients.

Our scheme is based on the construction of an effective Hamiltonian from first-principles calculations. A ferroelectric effective Hamiltonian 10 must include the ferroelectric local soft mode and the strain variables, since ferroelectric transitions are accompanied by a softening of a polar phonon mode and by the appearance of a strain. An alloy effective Hamiltonian must also include the compositional degrees of freedom. We propose to incorporate all such degrees of freedom by writing the total energy $E$ as a sum of two energies,

$$
E\left(\left\{\mathbf{u}_{i}\right\},\left\{\mathbf{v}_{i}\right\}, \eta_{H},\left\{\sigma_{j}\right\}\right)=E_{\text {ave }}\left(\left\{\mathbf{u}_{i}\right\},\left\{\mathbf{v}_{i}\right\}, \eta_{H}\right)
$$




$$
+E_{\mathrm{loc}}\left(\left\{\mathbf{u}_{i}\right\},\left\{\mathbf{v}_{i}\right\},\left\{\sigma_{j}\right\}\right),
$$

where $\mathbf{u}_{i}$ is the local soft mode in unit cell $i ;\left\{\mathbf{v}_{i}\right\}$ are the dimensionless local displacements which are related to the inhomogeneous strain variables inside each cell [10]; $\eta_{H}$ is the homogeneous strain tensor; and the $\left\{\sigma_{j}\right\}$ characterize the atomic configuration of the alloy. That is, $\sigma_{j}=+1$ or -1 corresponds to the presence of a $\mathrm{B}^{\prime}$ or $\mathrm{B}^{\prime \prime}$ atom, respectively, at lattice site $j$ of the $\mathrm{A}\left(\mathrm{B}^{\prime}{ }_{1-x} \mathrm{~B}^{\prime \prime}{ }_{x}\right) \mathrm{O}_{3}$ alloy. The energy $E_{\text {ave }}$ depends only on the soft mode and strain variables. The $\left\{\sigma_{j}\right\}$ parameters are thus incorporated into the second energy term $E_{\mathrm{loc}}$.

For $E_{\text {ave }}$, we generalize the analytical expression successfully used in Ref. [10 for simple $\mathrm{ABO}_{3}$ systems to the case of an alloy. This generalization simply consists in using the virtual crystal approximation (VCA) [11], i.e., in replacing $\mathrm{A}\left(\mathrm{B}^{\prime}{ }_{1-x}, \mathrm{~B}^{\prime \prime}{ }_{x}\right) \mathrm{O}_{3}$ by a uniform but composition-dependent "virtual" $\mathrm{ABO}_{3}$ system. $E_{\text {ave }}$ thus consists of five parts: a local-mode self-energy, a long-range dipole-dipole interaction, a short range interaction between soft modes, an elastic energy, and an interaction between the local modes and local strain [10].

For $E_{\text {loc }}$, we propose an expression that includes (i) the onsite effect of alloying on the self-energy up to the fourth order in the local-mode vector $\mathbf{u}_{i}$; and (ii) intersite contributions which are linear in $\mathbf{u}_{i}$ and in $\mathbf{v}_{i}$ :

$$
\begin{aligned}
& E_{\mathrm{loc}}\left(\left\{\mathbf{u}_{i}\right\},\left\{\mathbf{v}_{i}\right\},\left\{\sigma_{j}\right\}\right)= \\
& \quad \sum_{i}\left[\Delta \alpha\left(\sigma_{i}\right) u_{i}^{4}+\Delta \gamma\left(\sigma_{i}\right)\left(u_{i x}^{2} u_{i y}^{2}+u_{i y}^{2} u_{i z}^{2}+u_{i z}^{2} u_{i x}^{2}\right)\right] \\
& \left.\quad+\sum_{i j}\left[Q_{j, i}\left(\sigma_{j}\right) \mathbf{e}_{j i} \cdot \mathbf{u}_{i}+R_{j, i}\left(\sigma_{j}\right) \mathbf{f}_{j i} \cdot \mathbf{v}_{i}\right)\right]
\end{aligned}
$$

where the sums over $i$ and $j$ run over unit cells and mixed sublattice sites, respectively. $\Delta \alpha\left(\sigma_{i}\right)$ and $\Delta \gamma\left(\sigma_{i}\right)$ characterize the onsite contribution of alloying, while $Q_{j, i}\left(\sigma_{j}\right)$ and $R_{j, i}\left(\sigma_{j}\right)$ are related to alloying-induced intersite interactions. Here $\mathbf{e}_{j i}$ is a unit vector joining the site $j$ to the center of the soft-mode vector $\mathbf{u}_{i}$, and $\mathbf{f}_{j i}$ is a unit vector joining the site $j$ to the origin of the displacement $\mathbf{v}_{i}$. In principle, terms involving higher powers of $\mathbf{u}_{i}$ and $\mathbf{v}_{i}$ might be included to improve the quality of the expansion, but as will be shown below, we find this level of expansion to give a very good account of experimental findings. We also find that $Q_{j, i}\left(\sigma_{j}\right)$ and $R_{j, i}\left(\sigma_{j}\right)$ decrease rapidly as the distance between $i$ and $j$ increases. As a result, we included contributions up to the third neighbors for $Q_{j, i}\left(\sigma_{j}\right)$, and over the first-neighbor shell for $R_{j, i}\left(\sigma_{j}\right)$.

All the parameters of Eqs. (1) and (2) are derived from first principles. The 18 parameters of $E_{\text {ave }}$ (see Table II of Ref. 10 ) are determined by fitting the results of firstprinciples VCA calculations. On the other hand, $\Delta \alpha\left(\sigma_{i}\right)$, $\Delta \gamma\left(\sigma_{i}\right), Q_{j, i}\left(\sigma_{j}\right)$ and $R_{j, i}\left(\sigma_{j}\right)$ are derived by performing first-principles calculations in which a true atom [e.g., Ti or $\mathrm{Zr}$ in $\left.\mathrm{Pb}(\mathrm{Zr}, \mathrm{Ti}) \mathrm{O}_{3}\right]$ is surrounded by VCA atoms. The first-principles method used in the present study is the plane-wave ultrasoft-pseudopotential method [12] within the local-density approximation (LDA) [13]. The VCA

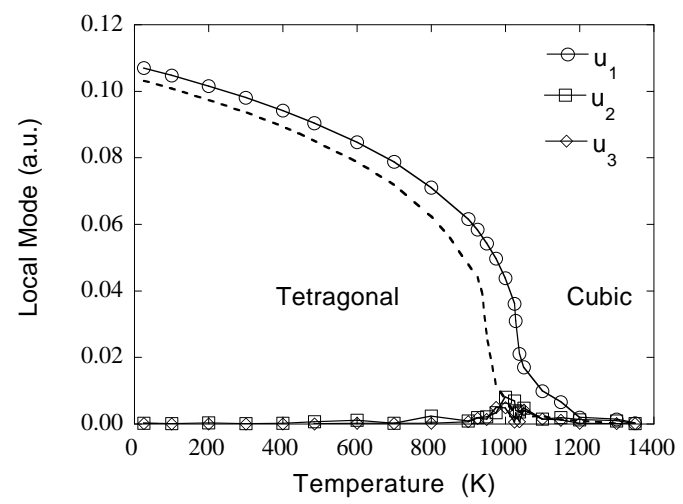

FIG. 1. Largest, middle, and smallest average cartesian coordinates $u_{1}, u_{2}$ and $u_{3}$ of the local-mode vector as a function of temperature in disordered single crystals of $\mathrm{Pb}\left(\mathrm{Zr}_{0.5} \mathrm{Ti}_{0.5}\right) \mathrm{O}_{3}$. Solid lines denote inclusion, while dashed li nes denote neglect, of $E_{\text {loc }}$ in Eq. (1). For clarity, $u_{2}$ and $u_{3}$ are not shown for the latter (VCA) case; they are nearly zero at all temperatures.

approach adopted averages the $\mathrm{B}^{\prime}$ and $\mathrm{B}^{\prime \prime}$ pseudopotentials, and is the one of Ref. [14].

Once our effective Hamiltonian is fully specified, the total energy of Eq. (1) is used in Monte-Carlo simulations to compute finite-temperature properties of ferroelectric alloys. We typically use a $12 \times 12 \times 12$ supercell (8640 atoms), since this choice yields well-converged results. The $\left\{\sigma_{j}\right\}$ variables are chosen randomly in order to mimic maximal compositional disorder - consistent with experimental reality [15]- and are kept fixed during the Monte-Carlo simulations. We find that averaging our results over a couple of different realizations of the disorder leads to well-converged statistical properties. The outputs of the Monte-Carlo procedure are the local mode vectors $\mathbf{u}$ (directly related to the electrical polarization), and the homogeneous strain tensor $\eta_{H}$. We use the correlation-function approach of Ref. [16] to derive the piezoelectric response from these Monte-carlo simulations. Up to $10^{6}$ Monte-Carlo sweeps are first performed to equilibrate the system, and then $2 \times 10^{4}$ sweeps are used to get the various statistical averages. The temperature is decreased in small steps.

Figure 1 shows the largest, middle and smallest cartesian coordinates $\left(u_{1}, u_{2}\right.$ and $\left.u_{3}\right)$ of the supercell average of the local mode vectors in $\mathrm{Pb}\left(\mathrm{Zr}_{0.5} \mathrm{Ti}_{0.5}\right) \mathrm{O}_{3}$ as a function of the temperature, as predicted by our approach described by Eqs. (1) and (2). Each coordinate is close to zero at high temperature, characterizing a paraelectric cubic phase. As the system is cooled down, $u_{1}$ drastically increases while $u_{2}$ and $u_{3}$ remain nearly null. This indicates a transition to a ferroelectric tetragonal phase, consistent with measurements [3]. We predict that the spontaneous polarization reaches $0.79 \mathrm{C} / \mathrm{m}^{2}$ at very low temperature, which compares well with the first-principles results of 0.70 and $0.74 \mathrm{C} / \mathrm{m}^{2}$ [5]. The tetragonal axial ratio $c / a$ ranges from 1 close to the transition region 


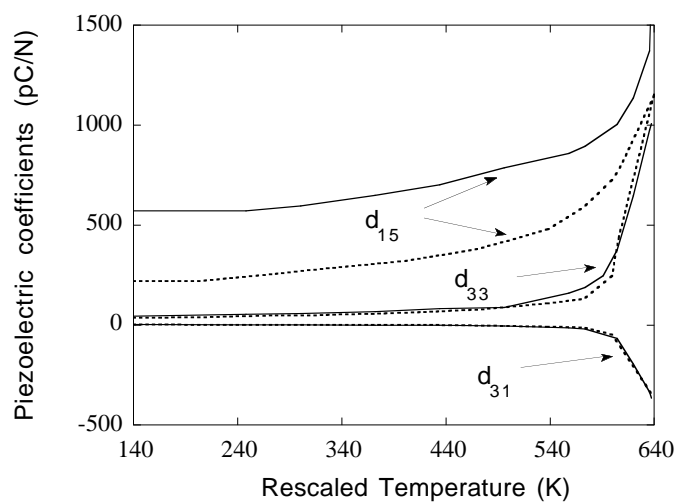

FIG. 2. Piezoelectric coefficients as a function of temperature in disordered $(4 \mathrm{~mm}) \mathrm{Pb}\left(\mathrm{Zr}_{0.5} \mathrm{Ti}_{0.5}\right) \mathrm{O}_{3}$. Solid lines denote inclusion, while dashed lines denote neglect, of $E_{\text {loc }}$ in Eq. (1). Temperature has been rescaled to fit the experimental value of the Curie temperature. Statistical errors are estimated to be $\sim 10 \%$ of the values displayed.

to 1.02 for lower temperature. This is in good agreement both with the experimental value of $1.02-1.025$ 3,7] obtained for disordered samples, and with the firstprinciples result of 1.03 obtained for an ordered alloy [6]. Figure 1 also shows the predictions of the VCA alloy theory, corresponding to the neglect of $E_{\mathrm{loc}}$ in Eq. (1). Interestingly, one sees that $E_{\text {loc }}$ has no effect on the phase transition sequence, which is consistent with recent findings that the VCA approach can reproduce some structural properties of PZT 14,17. Whether or not $E_{\text {loc }}$ is included in the total energy, we find a Curie temperature $T_{c}$ that is higher than the experimental value of $640 \mathrm{~K}$ 18. This difficulty of reproducing $T_{c}$ is a general feature of the effective-Hamiltonian approach [10,19,20], and may be due to higher perturbative terms neglected in the analytical expression for the total energy. In order to compare our results with experimental data, we will henceforth rescale our temperature as in Ref. [16] so that the theoretical $T_{c}$ is forced to match the experimental one.

Figure 2 shows the piezoelectric coefficients predicted for a tetragonal single crystal of $\mathrm{Pb}\left(\mathrm{Zr}_{0.5} \mathrm{Ti}_{0.5}\right) \mathrm{O}_{3}$ as a function of the rescaled temperature, when neglecting or incorporating $E_{\mathrm{loc}}$ in Eq. (1). The independent coefficients for the $4 \mathrm{~mm}$ point group are $d_{33}, d_{31}$ and $d_{15}$. One can notice that inclusion of $E_{\text {loc }}$ has only a small effect on $d_{31}$ and $d_{33}: d_{31}$ is rather small for any temperature except near the transition, and $d_{33}$ is around $50-$ $55 \mathrm{pC} / \mathrm{N}$ at room temperature in both simulations. Using the experimental values of the elastic compliances [5] to compute $e_{33}$ from our calculated $d_{31}$ and $d_{33}$, we find that our alloy effective hamiltonian leads to an $e_{33}$ of 4.3 $\mathrm{C} / \mathrm{m}^{2}$, while neglecting $E_{\text {loc }}$ in Eq. (1) yields a similar $e_{33}$ of $3.8 \mathrm{C} / \mathrm{m}^{2}$ at low temperature. Both predictions agree well with the first-principles results ranging between 3.4 and $4.8 \mathrm{C} / \mathrm{m}^{2}$ [5, 6, 14, confirming that the VCA can reproduce the $e_{33}$ coefficient of PZT [14].

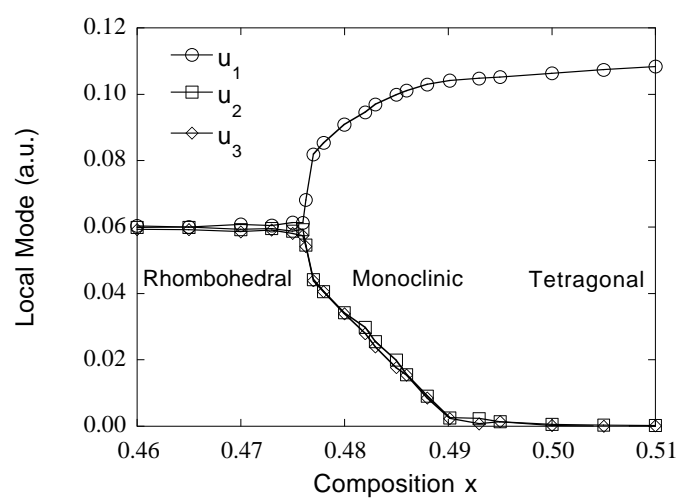

FIG. 3. Similar to Fig. (1), but computed as a function of composition $x$. The temperature of the simulation is $50 \mathrm{~K}$, corresponding to a rescaled experimental value of $30 \mathrm{~K}$ (see text).

Figure 2 also demonstrates that incorporating $E_{\text {loc }}$ in the total energy leads to a large enhancement of the $d_{15}$ coefficient, which is consistent with recent measurements revealing that the piezoelectric elongation of the tetragonal unit cell of PZT does not occur along the polar direction [21]. This enhancement is highly relevant for the piezoelectric response $d_{33}$ in ceramic samples, denoted $d_{33, c}$, which involves an average of the form

$$
d_{33, c}=\int_{0}^{\pi / 2}\left[\left(d_{31}+d_{15}\right) \sin ^{2} \theta+d_{33} \cos ^{2} \theta\right] \sin \theta \cos \theta d \theta
$$

over the single-crystal coefficients. The true alloy approach of Eqs. (1)-(2) leads to a $d_{33, c}$ of $163 \mathrm{pC} / \mathrm{N}$ at room temperature, in excellent agreement with the experimental value of $170 \mathrm{pC} / \mathrm{N}$ [4] 22]. On the other hand, neglecting $E_{\text {loc }}$ leads to a smaller $d_{33, c}$ of $90 \mathrm{pC} / \mathrm{N}$. This difference clearly demonstrates the necessity of incorporating the local alloying effect into the total energy for understanding the large piezoelectric response of PZT ceramics near the $\mathrm{MPB}$.

We now use our alloy effective Hamiltonian to investigate the low-temperature phases of $\mathrm{Pb}\left(\mathrm{Zr}_{1-x} \mathrm{Ti}_{x}\right) \mathrm{O}_{3}$ near the MPB. We choose a constant temperature of 50 $\mathrm{K}$ in the Monte-Carlo simulations, and vary the overall composition of the solid solution. This compositional variation affects two quantities: (i) the populations of $\sigma_{j}$ equal to +1 or -1 , and (ii) the alloy-related parameters. For the latter, only the parameters entering the local-mode self-energy of $E_{\text {ave }}$ 10, and the $\Delta \alpha\left(\sigma_{i}\right)$ and $\Delta \gamma\left(\sigma_{i}\right)$ in Eq. (2), are allowed to be compositiondependent. This composition-dependence is assumed to be linear, and is determined by performing first-principles simulations on two different compositional cells. Such a linear composition-dependence approach is only realistic when exploring a narrow range of compositions, as done in the present study.

Figure 3 shows that the local mode, and hence the po- 
larization, is parallel to the pseudo-cubic [001] direction for Ti compositions larger than $50 \%$, which is consistent with a tetragonal phase. For compositions lower than $47 \%$, the polarization becomes parallel to the pseudocubic [111] direction, indicating the "high temperature" rhombohedral phase [3,23]. The most interesting feature of Fig. 3 is the behavior of the local mode for the compositional range between $47.5 \%$ and $49.5 \%$ : as $x$ decreases, $u_{1}$ decreases, while $u_{2}$ and $u_{3}$ increase and remain nearly equal to each other. This behavior is characteristic of an intermediate phase that is neither tetragonal nor rhombohedral. The strain tensor given by our simulations indicates that this intermediate phase is the monoclinic phase experimentally found by Noheda et al. We further predict that the monoclinic phase for $x \simeq 48 \%$ can be characterized by an angle of $90.7^{\circ}$ and by lattice vectors $\mathbf{a}_{m}=a_{0}(-1.005,-1.005,-0.009), \mathbf{b}_{m}=$ $a_{0}(1.002,-1.002,0.000)$, and $\mathbf{c}_{m}=a_{0}(0.004,0.004,1.018)$, where $\mathrm{a}_{0}$ is a cubic lattice constant. All these predictions are in excellent quantitative agreement with the experimental results of Ref. [7]. Figure 3 clearly demonstrates that the monoclinic phase acts as a bridge between the rhombohedral and tetragonal phases, as indicated by the continuous rotation of the polarization as a function of composition. Our computational scheme is also able to reproduce the compositional range narrowing of the monoclinic phase observed when increasing the temperature [21]. It should be noted that a VCA-only calculation (i.e., neglecting $E_{\mathrm{loc}}$ ) does not reveal a monoclinic phase. This finding demonstrates once again the need for incorporating the local effect of alloying into the total energy to study subtle effects.

In summary, we have developed a first-principles derived computational scheme to study finite-temperature properties of $\mathrm{Pb}\left(\mathrm{Zr}_{1-x} \mathrm{Ti}_{x}\right) \mathrm{O}_{3}$ solid solutions near the $\mathrm{MPB}$ as a function of composition and temperature. We find that there is a low-temperature monoclinic phase acting as a bridge between the rhombohedral phase, existing for $x<0.47$, and the tetragonal phase, occurring for $x$ larger than 0.50 . The predicted structural data are in very good agreement with measurements, as well as with direct first-principles calculations. The use of this approach also provides an explanation for the large experimental value of $d_{33}$ in tetragonal ceramics of PZT near the MPB 221. This large piezoelectricity is simply due to the very large value of the $d_{15}$ coefficient predicted to occur in the single crystal.

L.B. thanks the financial assistance provided by the Arkansas Science and Technology Authority (grant N99B-21), and the National Science Foundation (grant DMR-9983678). A.G. acknowledges support from the Spanish Ministry of Education (grant PB97-0598). D.V. acknowledges the financial support of Office of Naval Research grant N00014-97-1-0048. We wish to thank B. Noheda, H. Chen, M. Cohen, E. Cross, T. Egami and Q. Zhang for very useful discussions.
[1] K. Uchino, Piezoelectric actuators and ultrasonic motors, (Kluwer Academic Publishers, Boston) (1996).

[2] M.A. Akbas and P.K. Davies, J. Am. Ceram. Soc. 81, 670 (1998)

[3] M.E. Lines and A.M. Glass, Principles and Applications of Ferroelectrics and Related Materials (Clarendon Press, Oxford, 1977).

[4] X.-H. Du, U. Belegundu and K. Uchino, Jpn. J. Appl. Phys. 36, 5587 (1997); X.-H. Du, J. Zheng, U. Belegundu and K. Uchino, Appl. Phys. Lett. 72, 2421 (1987).

[5] G. Saghi-Szabo, R.E. Cohen and H. Krakauer, Phys. Rev. $B$ 59, 12771 (1999).

[6] L. Bellaiche and D. Vanderbilt, Phys. Rev. Lett., 83, 1347 (1999).

[7] B. Noheda, D.E. Cox, G. Shirane, J.A. Gonzalo, L.E. Cross, and S-E. Park, Appl. Phys. Lett. 74, 2059 (1999).

[8] D. Vanderbilt, Curr. Opin. Solid State Mater. Sci., 2, 701 (1997).

[9] L. Bellaiche, J. Padilla and David Vanderbilt, Phys. Rev. B. 59, 1834 (1999).

[10] W. Zhong, D. Vanderbilt and K.M. Rabe, Phys. Rev. Lett. 73, 1861 (1994); Phys. Rev. B 52, 6301 (1995);

[11] L. Nordheim, Ann. Phys. (Leipzig) 9, 607 (1931).

[12] D. Vanderbilt, Phys. Rev. B 41, 7892 (1990).

[13] P. Hohenberg and W. Kohn, Phys. Rev. 136, B864 (1964); W. Kohn and L.J. Sham, ibid. 140, A1133 (1965).

[14] L. Bellaiche and D. Vanderbilt, Phys. Rev. B 61, 7877 (2000).

[15] L.E. Cross, Ferroelectrics 151, 305 (1994).

[16] A. García and D. Vanderbilt, in First-Principles Calculations for Ferroelectrics: Fifth Williamsburg Workshop, R.E. Cohen, ed. (AIP, Woodbury, New York, 1998), p. 53; Appl. Phys. Lett. 72, 2981 (1998).

[17] N. J. Ramer and A. M. Rappe, J. Phys. Chem. Solids, 61, 317 (2000).

[18] T. Yamamoto, Jpn. J. Appl. Phys. 35, 5104 (1996).

[19] U. Waghmare and K. Rabe, Phys. Rev. B 55, 6161 (1997).

[20] H. Krakauer, R. Yu, C.-Z. Wang and C. LaSota, Ferroelectrics 206-207, 133 (1998).

[21] R. Guo, L.E. Cross, S-E. Park, B. Noheda, D.E. Cox and G. Shirane, cond-mat., 9912118 (1999).

[22] D.A. Berlincourt, C. Cmolik and H. Jaffe, Proc IRE 48, 220 (1960).

[23] Our model cannot predict the "low-temperature" rhombohedral phase 3] since this involves octahedra-tilting degrees of freedom that are not including in the present model. Our simulations thus also demonstrate that the oxygen tilts are not the driving force for the transition or for the occurrence of a monoclinic phase. 